\title{
Medicolegal responsibilities for the administration of intravenous contrast media by radiographers: Radiologists' perspectives
}

\author{
G G V Koch, ${ }^{1}$ MHSc Radiogr (Diagn), PGDip Tertiary Educ; L D Swindon, ${ }^{1}$ MEd (Higher Ed), BTech Radiogr (Diagn); \\ J D Pillay, ${ }^{2}$ PhD Physiol, MPH \\ 1 Department of Radiography, Faculty of Health Sciences, Durban University of Technology, South Africa \\ ${ }^{2}$ Department of Basic Medical Sciences, Faculty of Health Sciences, Durban University of Technology, South Africa
}

Corresponding author: G GV Koch (erhardkoch9@gmail.com)

\begin{abstract}
Background. Global trends in the delivery of healthcare services have placed tremendous strain on resources, among them human and capital. With this has emerged the need to revisit the job requirements and/or scope of practice of cadres within a profession, to ensure adequate training where needed. The administration of intravenous contrast media (IVCM), a fundamental element of expertise within the radiology field, is an example of such evolution in South Africa (SA). Currently falling within radiologists' scope of practice, it has become necessary for radiographers to extend their own scope to include this skill, owing to the national shortage of radiologists and subsequent service delivery constraints, as well as the need to close the gap with international trends.

Objective. To provide a synopsis of the perspectives of radiologists on the medicolegal responsibilities related to the administration of IVCM by radiographers.

Methods. A quantitative, descriptive, cross-sectional research design was conducted, targeting qualified radiologists in KwaZulu-Natal Province (KZN). An online questionnaire was administered through SurveyMonkey that provided information on the medicolegal responsibilities associated with the administration of IVCM.

Results. Of a total of 97 qualified radiologists in $\mathrm{KZN}$, a response rate of $48.5 \%$ ( $n=47)$ was obtained. The majority of respondents felt that radiographers should be responsible for obtaining informed patient consent (66.0\%), and deciding on the site of IVCM administration (72.3\%). It was also felt that the radiologists should remain responsible for decisions regarding the type and dose of IVCM (87.2\%) and managing the possible complications and adverse reactions due to the administration of IVCM (78.7\%).

Conclusion. Evidence-based research provides a contextualised approach towards addressing transformation in service delivery and training needs. This study, in demonstrating the importance of appropriate medicolegal responsibilities in the extension of a professional role, forms a basis for informing the future training of radiographers in SA.
\end{abstract}

S Afr J Bioethics Law 2018;11(2):60-65. DOI:10.7196/SAJBL.2018.v11i2.632

Role extension can be defined as the adoption of a role or responsibility that did not previously form part of one's original roles or responsibilities. ${ }^{[1]} \mathrm{Global}$ trends in the delivery of healthcare services have warranted the need to revisit the job requirements and/ or scope of practice of cadres within professions, and to engage in possible role extension. ${ }^{[2]}$ The administration of intravenous contrast media (IVCM), a fundamental area of expertise within the radiology field, is an example of such a requirement. The administration of IVCM is currently under consideration by the Professional Board for Radiography and Clinical Technology (PBRCT) for inclusion as an extension of the South African (SA) radiographer's role..$^{[1,3,4]}$ In so doing, it is important to identify the medicolegal responsibilities that should become part of the radiographer's scope of practice, and those that should remain within the radiologists' scope, to ensure clarity and harmony between the two professions.

According to annexure 10 of the Ethical Rules of Conduct for Practitioners registered under the Health Professions Act No. 56 of
1974, SA radiographers may not perform tasks outside the scope of their training ${ }^{[5]}$ They are not permitted to administer IVCM, because they have not received the necessary accredited training to do so. Furthermore, it is not within the scope of the radiography profession. However, in a recent study conducted by the PBRCT, $44.2 \%$ of respondents (radiographers) indicated that they are currently administering IVCM. This is a violation of the regulations defining the scope of profession of radiography and can have serious professional and medicolegal consequences. ${ }^{[6]}$

In a position statement published in 2011 by the Radiological Society of SA (RSSA), radiologists in SA do support the idea of radiographers administering IVCM, in principle, provided that the necessary PBRCT-approved and accredited training is obtained..$^{[7]}$ This is largely driven by: (i) the national shortage of radiologists, and the subsequent service delivery constraints; and (ii) the gap between $\mathrm{SA}$ and international trends. ${ }^{[1,7]}$ Radiographers practising in the UK, Ireland, USA, Europe, Canada and Australia have been able to extend 
their professional roles to include the administration of IVCM..$^{[-13]}$ Each of these countries, however, has different regulatory frameworks, policies and procedures pertaining to the medicolegal responsibilities of radiographers administering IVCM. It is, therefore, reasonable to justify the argument that evidence-based research would be a suitable approach to addressing local healthcare needs. Consequently, the input of radiologists regarding this role extension would be the most relevant, as the administration of IVCM falls within their current scope of practice.

The chairperson of the current PBRCT confirmed that minimum training standards for the administration of IVCM by radiographers are currently being drafted by the PBRCT (personal communication, 28 July 2017). This study set out to identify the medicolegal responsibilities that are unique to the SA radiographer in the local context. The specific areas of medicolegal responsibilities identified in this study were: (i) obtaining informed patient consent; (ii) deciding on the type and dose of IVCM to be administered; (iii) deciding on the site of IVCM administration; and (iv) the overall responsibility for managing the possible complications and adverse reactions that may occur.

\section{Objective}

To provide a synopsis of the perspectives of radiologists on the medicolegal responsibilities related to the administration of IVCM by radiographers.

\section{Methods \\ Research design}

The present study used a quantitative, descriptive, cross-sectional research design.

\section{Study setting and target population}

This study was conducted in KwaZulu-Natal Province (KZN), SA, and targeted qualified radiologists registered with the Health Professions Council of SA (HPCSA). Radiologists were targeted because the administration of IVCM currently falls within their scope of profession, and they, therefore, have knowledge of the appropriate training required.

\section{Sample selection and sampling technique}

The study sample (radiologists) was identified by means of the HPCSA's iRegister, an online database representing all those health professionals who have previously registered with the HPCSA. The iRegister at the time revealed 104 radiologists in KZN. Seven of these were identified as being deceased, resulting in a more accurate sample of 97 radiologists. A purposive, non-probability sampling technique based on the nature of the research study was used. ${ }^{[18]}$ All radiologists working in KZN who met the study criteria were included. The inclusion criteria were qualified radiologists registered with the HPCSA under the specialty of diagnostic radiology who were either living and practising in KZN (in the private and/or public sectors), or who had retired after doing so.

\section{Research tool and data analysis}

An online questionnaire administered through SurveyMonkey was used, via email communication. The research tool consisted of closed- and open-ended questions and statements pertaining to the medicolegal responsibilities associated with radiographers administering IVCM. The variables collected included radiologists' opinions on who should be responsible for: (i) obtaining informed patient consent; (ii) deciding on the type and dose of IVCM to be administered; (iii) deciding on the site of IVCM administration; and (iv) the overall responsibility for managing possible complications and adverse reactions.

Primary data were collected and analysed using version 23.0 of the Statistical Package for the Social Sciences (IBM Corp., USA). Descriptive and inferential statistics were used for the analysis; quantitative data were summarised and presented in the form of tables and figures. The data were grouped according to the respondents' ages and employment sectors, identifying common themes and possible differences between the perspectives of the older and younger radiologists in both the private and public sectors. The Pearson $\chi^{2}$ and Cronbach's $\alpha$ tests were performed for statistical significance and for reliability of the research tool, respectively. ${ }^{[14,15]}$

\section{Focus-group discussion and data-collection process}

A focus-group discussion was held prior to the data-collection process to ensure reliability and validity of the content of the research tool. ${ }^{[16]}$ This involved a critique of the questionnaire by one radiologist, two radiographers, the researcher and the research supervisors. The study participants were contacted in their personal capacity by means of email and/or social media. The RSSA used their official Facebook page to encourage their KZN members to participate in the study. The data were collected over the course of 3 months, and stored in password-protected files by the researcher.

\section{Ethical considerations}

Ethical approval for conducting this study was obtained from the Institutional Research Ethics Committee at the Durban University of Technology (ref. no. REC 18/15). Informed consent was provided by all participants and those in the focus group, through completion of the online questionnaire and informed consent forms, respectively.

\section{Results}

Of a total of 97 qualified radiologists in $\mathrm{KZN}$, a response rate of $48.5 \%(n=47)$ was obtained. The majority of the respondents were employed exclusively in the private sector $(68.1 \% ; n=32)$ as opposed to exclusively in the public sector $(23.4 \% ; n=11)$. The remainder were employed in both the private and public sectors (8.5\%) $(n=4)$. In this article, the focus is directed to the differences of opinion between the exclusively private and public sector respondents.

\section{Informed patient consent}

Overall, $66.0 \%$ ( $n=31$ ) of the respondents believed that radiographers should be responsible for obtaining informed patient consent, whereas $19.1 \%(n=9)$ felt that it should be the responsibility of radiologists and $14.9 \%(n=7)$ indicated 'other' (Table 1). Among the 'other' responses, the following were identified as suggestions: (i) both the radiographer and radiologist; (ii) the radiographer, radiologist and referring practitioner; or (iii) the reception staff should be responsible for obtaining informed consent. From an employment sector perspective, $75.0 \%$ of the private sector respondents felt that radiographers should be responsible for obtaining informed 
consent, whereas most public sector respondents (45.4\%) felt that the radiologist should remain responsible (Fig. 1).

\section{Type and dose of intravenous contrast media}

The majority of the respondents $(87.2 \% ; n=41)$ felt that the decision regarding the type and dose of IVCM to be administered should be the responsibility of the radiologist, whereas $10.6 \%(n=5)$ felt that it should be the radiographer's responsibility. One of the respondents in the 50 - 59-year age group felt that it should be a joint responsibility between the radiographer, radiologist and referring practitioner (Table 1). Both the private and public sector respondents $(\geq 75 \%)$ seemed to agree that radiologists should remain responsible for deciding on the type and dose of IVCM to be administered (Fig. 2).

\section{Site of administration}

The decision on the site of IVCM administration was agreed upon by the majority of the respondents $(72.3 \% ; n=34)$ to be the responsibility of the radiographer administering the IVCM. This contrasts with the $21.3 \%(n=10)$ who felt that it should be the responsibility of the radiologist (Table 1). The private sector radiologists seemed to be

Table 1. View of who should have medicolegal responsibility for the administration of IVCM by respondent age group, $\boldsymbol{n}$ (\%)

\begin{tabular}{|c|c|c|c|c|c|}
\hline \multirow[b]{2}{*}{ Medicolegal responsibility } & \multicolumn{4}{|c|}{ Age group } & \multirow[b]{2}{*}{ Total $(N=47)$} \\
\hline & $30-39$ & $40-49$ & $50-59$ & $60-69$ & \\
\hline \multicolumn{6}{|l|}{ Informed consent } \\
\hline Radiographer & $3(50.0)$ & $18(75.0)$ & $8(53.3)$ & $2(100.0)$ & $31(66.0)$ \\
\hline Radiologist & $2(33.3)$ & $4(16.7)$ & $3(20.0)$ & $0(0.0)$ & $9(19.0)$ \\
\hline Other & $1(16.7)$ & $2(8.3)$ & $4(26.7)$ & $0(0.0)$ & $7(15.0)$ \\
\hline Total & $6(13.0)$ & $24(51.0)$ & $15(32.0)$ & $2(4.0)$ & \\
\hline \multicolumn{6}{|l|}{ Type and dose } \\
\hline Radiographer & $1(16.7)$ & $3(12.5)$ & $1(6.7)$ & $0(0.0)$ & $5(11.0)$ \\
\hline Radiologist & $5(83.3)$ & $21(87.5)$ & $13(86.7)$ & $2(100.0)$ & $41(87.0)$ \\
\hline Other & $0(0.0)$ & $0(0.0)$ & $1(6.7)$ & $0(0.0)$ & $1(2.0)$ \\
\hline Total & $6(13.0)$ & $24(51.0)$ & $15(32.0)$ & $2(4.0)$ & \\
\hline \multicolumn{6}{|l|}{ Site of administration } \\
\hline Radiographer & $2(33.3)$ & $20(83.3)$ & $10(66.7)$ & $2(100.0)$ & $34(73.0)$ \\
\hline Radiologist & $3(50.0)$ & $4(16.7)$ & $3(20.0)$ & $0(0.0)$ & $10(21.0)$ \\
\hline Other & $1(16.7)$ & $0(0.0)$ & $2(13.3)$ & $0(0.0)$ & $3(6.0)$ \\
\hline Total & $6(13.0)$ & $24(51.0)$ & $15(32.0)$ & $2(4.0)$ & \\
\hline \multicolumn{6}{|c|}{ Complications and adverse reactions } \\
\hline Radiographer & $0(0.0)$ & $3(12.5)$ & $0(0.0)$ & $0(0.0)$ & $3(6.0)$ \\
\hline Radiologist & $5(83.3)$ & $18(75.0)$ & $12(80.0)$ & $2(100.0)$ & $37(79.0)$ \\
\hline Other & 1 (16.7) & $3(12.5)$ & $3(20.0)$ & $0(0.0)$ & $7(15.0)$ \\
\hline Total & $6(13.0)$ & $24(51.0)$ & $15(32.0)$ & $2(4.0)$ & \\
\hline
\end{tabular}

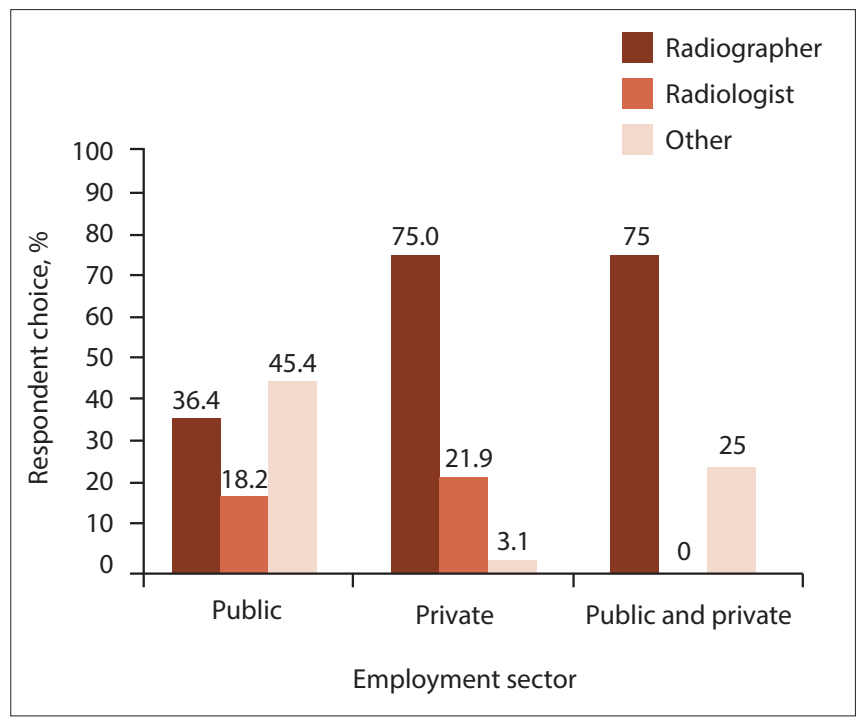

Fig. 1. Respondent choice as to who should have responsibility for obtaining informed patient consent for IVCM. (IVCM = intravenous contrast media.)

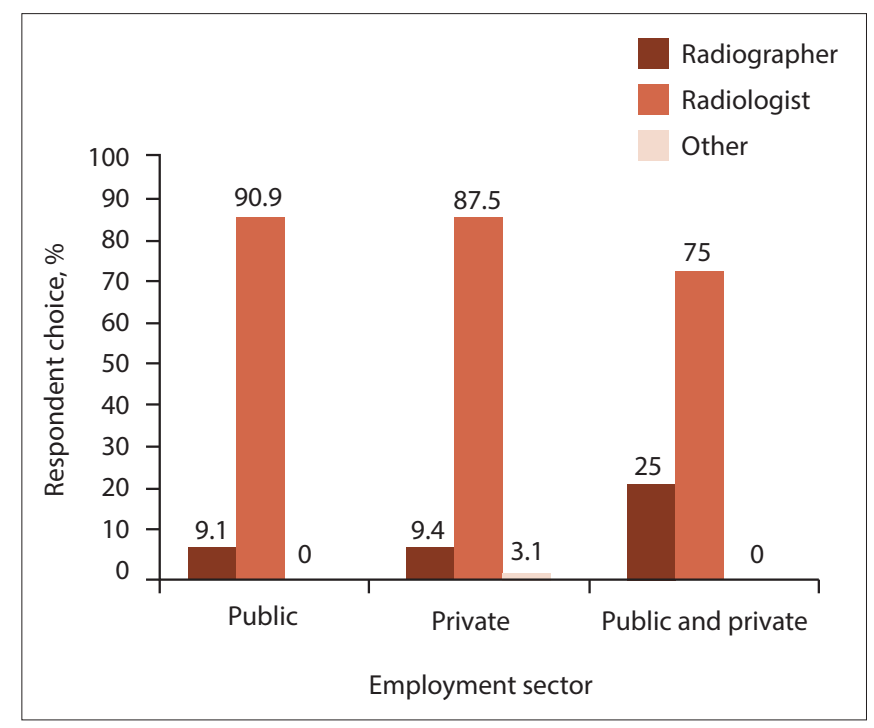

Fig. 2. Respondent choice as to who should decide on type and dose of IVCM. (IVCM = intravenous contrast media.) 
at a higher level of agreement on this (81.2\%), compared with the $54.5 \%$ of those in the public sector who felt that it should remain the responsibility of the radiologist (Fig. 3). Based on these results, there is a clear difference of opinion between the private and public sector radiologists on this issue.

\section{Complications and adverse reactions}

The majority of the respondents (78.7\%) $(n=37)$ believed that radiologists should remain responsible for managing the possible complications and adverse reactions that may occur after the administration of IVCM, and only $6.4 \%$ ( $n=3)$ felt that it should be the responsibility of the radiographer. Those respondents who were in favour of radiographers being responsible were in the 40 - 49-year age group (Table 1). From an employment sector perspective (Fig. 4), there was no doubt as to who should be responsible, as the public and private sectors both reached a consensus ( $>60 \%$ ) that radiologists should remain responsible for managing possible complications and adverse reactions.

\section{Discussion}

The roles and responsibilities of professionals can easily be misinterpreted and misrepresented. In the healthcare domain, this is often evident across different groups within specific professions; in the radiology profession, this is particularly evident between

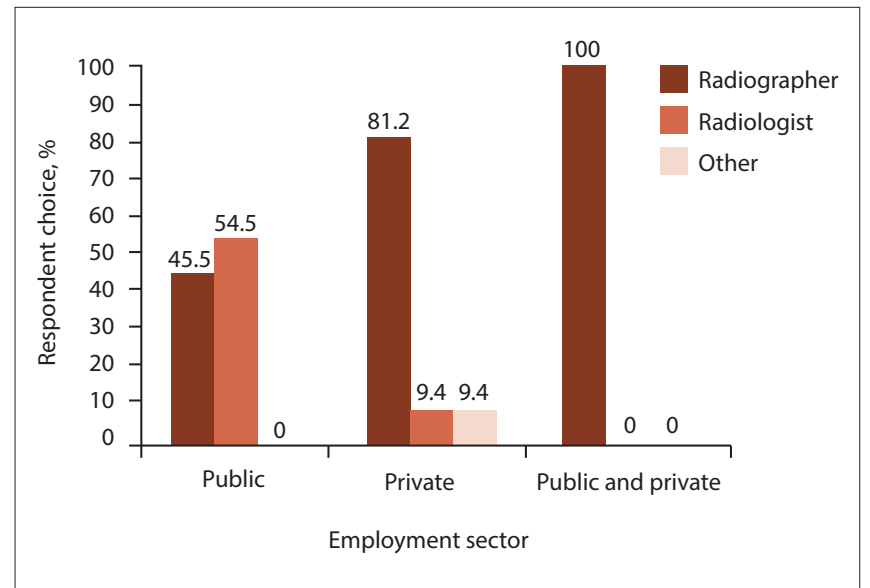

Fig. 3. Respondent choice as to who should decide on site of administration of IVCM. (IVCM = intravenous contrast media.)

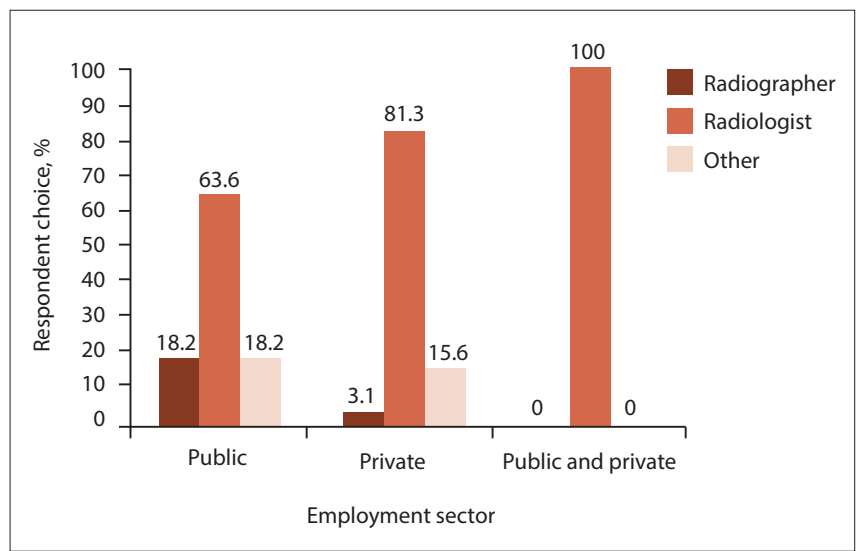

Fig. 4. Respondent choice as to who should have responsibility for managing possible complications of and adverse reactions to IVCM. (IVCM = intravenous contrast media.) radiographers and radiologists. ${ }^{[2]}$ These professionals work in close collaboration with each other, and their daily tasks may often overlap. Careful attention must, therefore, be paid to the medicolegal responsibilities associated with each profession, to prevent the possibility of litigation and to align with international practice relating to the extended roles of radiographers.

\section{Informed patient consent}

In the context of this study, informed patient consent refers to a patient giving permission to receive IVCM after the possible complications and adverse reactions have been clearly explained by a responsible and adequately trained professional. Informed consent thus allows a patient to make decisions regarding their own treatment and further management, while protecting their human rights. ${ }^{[17,18]}$ Medical law and legislation create a protective framework for the safety and welfare of patients. This protective framework ensures that patients are protected against medical malpractice. ${ }^{[19]}$

The HPCSA states that a patient has the right to make an informed decision regarding consent, and to be informed of the possible complications and adverse reactions related to a medical procedure. ${ }^{[20]}$ In the present study, the results show that it was generally felt that radiographers should be responsible for obtaining informed patient consent prior to administering IVCM, despite the minority of respondents who indicated that the reception staff should share the responsibility. It is important to keep in mind that the reception staff may not have received formal training in radiography or radiology, and do not have sufficient knowledge of the possible complications and adverse reactions of IVCM. They are therefore not in a position to be able to clearly explain the possible complications and adverse reactions that may occur due to the administration of IVCM.

In Australia, radiologists maintain the responsibility for obtaining informed patient consent, in contrast with the opinions indicated by the findings of this study. ${ }^{[13]}$ In the UK, it is the responsibility of the individual administering the IVCM to obtain informed patient consent. ${ }^{[2]}$ Based on the findings from the present study, it is safe to conclude that radiologists agree that whoever administers the IVCM (radiologist or radiographer) should be responsible for obtaining informed consent from the patient, as both professionals would be adequately trained to perform this function.

\section{Type and dose of intravenous contrast media}

The type and dose of IVCM directly affects the probability of complications and adverse reactions after it is administered. ${ }^{[22]}$ The type and dose depend on the age of the patient, his/her risk factors for allergies, the medical history and the type of examination. ${ }^{[23]}$ The radiologists who participated in this study believed that they should remain responsible for deciding on the type and dose of contrast, even if it is to be administered by a radiographer. Radiologists in Australia have also maintained this responsibility, as it falls under the prescribing of drugs, and are therefore in agreement with those in the present study ${ }^{[13]}$ This is a significant finding, in that the study respondents are in agreement with an existing, international model.

According to the literature, in SA, only those practitioners who are registered with the relevant professional council and whose premises have been licensed in terms of the Medicines and Related Substances Control Act No. 101 of 1965 may prescribe drugs. No person registered under the Health Professions Act No. 56 of 1974 
can prescribe a substance unless authorised by his/her professional council. ${ }^{[24]}$ Important to note is that currently, radiographers in SA are neither formally trained to manage or treat patients, nor to prescribe drugs, and would need extensive additional training in this regard to be legally compliant. Radiologists, however, are permitted to prescribe drugs, including IVCM.

\section{Site of administration}

There is a paucity of literature on the medicolegal responsibility associated with deciding on the site of IVCM administration. The risk of soft-tissue extravasation and infection, however, made it necessary to investigate this aspect, as radiologists have, in the past, been sued by their patients for injuries associated with the extravasation of IVCM. ${ }^{[25,26]}$ The results of the present study indicate that a radiographer who administers IVCM should also be responsible for deciding on the site of administration. Although the private sector respondents showed greater consensus than those in the public sector regarding this responsibility, there was a general agreement that the person administering the IVCM should be responsible for deciding on the site of administration, with the proviso that adequate training is obtained.

\section{Complications and adverse reactions}

The Medscape 2015 malpractice report ${ }^{[26]}$ revealed that in addition to failure to obtain informed patient consent, radiologists globally are often sued for the complications and adverse reactions associated with the administration of IVCM. This was supported by a recently published article that emphasised the readiness of radiologists to attend to a patient experiencing complications and adverse reactions. Although the composition of IVCM on the market today is better tolerated by many patients, the risk of complications and adverse reactions cannot be excluded. ${ }^{[22]}$

The present study found that even if the administration of IVCM becomes a part of the radiographers' scope of practice, radiologists should remain responsible for treating and managing the possible complications and adverse reactions. These recommendations are similar to and on par with the practice standards in the UK and Australia, according to which radiologists remain responsible for the patient's welfare. ${ }^{[3,13]}$ The reactions from IVCM may vary from mild to moderate and severe, and may even cause death. ${ }^{[27-29]}$ Should radiographers be held responsible for managing complications and adverse reactions, extensive additional training would be needed.

From these results, it appears that a radiologist or medical officer should be physically present during each case of IVCM administration. Although the risk of litigation for the radiographer may be considerably lower than that of the radiologist, formal training and malpractice insurance for radiographers are highly recommended. This conclusion was supported by the results of a local study that targeted radiographers. ${ }^{[3]}$

\section{Summary of study findings}

In summary, the medicolegal responsibilities for the administration of IVCM identified by the respondents are:

- Radiographers should be responsible for obtaining informed patient consent when administering IVCM.

- Radiologists should remain responsible for deciding on the type and dose of IVCM to be administered.
- Radiographers should be responsible for deciding on the site of IVCM administration in the patients that they attend to.

- Radiologists should remain responsible for managing any possible complications and adverse reactions resulting from the administration of IVCM. The authors therefore recommend that no radiographer should administer IVCM without a radiologist or medical officer being physically present in the department, even after having successfully completed the necessary accredited training to perform IVCM administrations.

\section{Implications of the findings}

The findings of this study may be useful to inform the future scope of profession of radiographers, and the associated training requirements for radiography in SA. The present study hopes to inform all healthcare professions of the importance of patient rights, medical law and the reality of medical malpractice litigation, especially in cases where professional roles are extended. Such an application is relevant to most professions, particularly those involving multi-leveled cadres with blurred levels of overlap in job requirements and/or scopes of practice between them.

\section{Study strengths}

- This study was, to the best of our knowledge, the first in SA to explore the medicolegal responsibilities of radiographers in administering IVCM, in anticipation of the extension of their professional role.

- This study obtained input from qualified radiologists whose scope of profession permits the administration of IVCM. They were therefore best equipped to provide reliable data for this study, and their input and support is extremely valuable.

\section{Study limitations}

- The study participants were KZN radiologists, the majority of whom were employed in the private sector. The private sector respondents' input may have outweighed the opinions of the public sector respondents, leaving a possible gap in the data.

- This study did not include a question on the physical presence of the radiologist or medical officer while the radiographer administers IVCM. The authors, however, have made an assumption in this regard that this is necessary.

\section{Recommendations}

It is recommended that this study be replicated at national level for a national consensus on the medicolegal responsibilities of radiographers in SA in administering IVCM. Future research should also include questions regarding the physical presence of the radiologist (or medical officer). Furthermore, input should be obtained from a larger portion of public sector radiologists.

\section{Conclusion}

Evidence-based research provides a contextualised approach to addressing local healthcare service delivery and training needs, while integrating international practice standards. This study has demonstrated the importance of identifying the appropriate medicolegal responsibilities associated with the extension of a professional role. It is hoped that this will inform future training for radiographers in SA through the valuable input obtained from radiologists. 
Acknowledgements. The first author would like to thank the RSSA for their assistance during the data collection process, the study respondents for participation in the study and the co-authors for their guidance in finalising this article.

Author contributions. GGVK and LDS contributed to the conceptualisation of this study; GGVK conducted the study and reported the findings; LDS and JDP provided support during the study as academic supervisors; GGVK, LDS and JDP contributed to the preparation of this manuscript.

Funding. Financial support for conducting this research study was received from the Durban University of Technology.

\section{Conflicts of interest. None.}

1. Gqweta N. Role extension: The needs, perceptions and experiences of South African radiographers in primary health care. S Afr Radiographer 2012;50(1):22-26

2. Koch GGV, Swindon LD, Pillay JD. Training requirements for the administration of intravenous contrast media by radiographers: Radiologists' perspective. Afr J Health Professions Educ 2017;9(3):128-132. https://doi.org/10.7196/AJHPE.2017. v9i3.809

3. Munro L, Isaacs F, Friedrich-Nel H, Swindon L. An analysis of the need for accredited training on the administration of intravenous contrast media by radiographers: results of an online survey. S Afr Radiographer 2012;50(2):27-34.

4. Kekana RM, Swindon LD, Mathobisa JM. A survey of South African radiographers' and radiologists' opinions on role extension for radiographers. Afr J Physical Health Educ Recreation Dance 2015;21(4.1):1114-1125.

5. Health Professions Council of South Africa. Ethical Rules of Conduct for Practitioners Registered under the Health Professions Act, 1974. Government Gazette 29079. 30 July. http://www.hpcsa.co.za/downloads/ethical_rules/ethical_rules_of_conduct_2011.pdf (accessed 26 September 2017).

6. Kekana RM, Swindon LD, Mathobisa JM. Results of the Radiography Role Extension Research Survey. http://www.hpcsa.co.za/PBRadiography/Guidelines (accessed 29 July 2017). Pretoria: HPCSA, 2014.

7. Radiological Society of South Africa. RSSA Position Statement on the Injection of Contrast. Cape Town: RSSA, 2011. http://rssa.co.za/downloads/doc_download/1240rssa-position-statement-on-the-injecting-of-contrast (accessed 27 September 2017).

8. Society and College of Radiographers. Course of Study for the Certification of Competence in Administering Intravenous Injections. London: SOR, 2011. http://www.sor.org/system/files/article/201202/SoR_IV_Document_proof3.pdf (accessed 29 July 2017).

9. Irish Institute of Radiography and Radiation Therapy. Intravenous Administration by Radiographers - Guidelines on Best Practice. 4th ed. Dublin: IIRRT, 2014. http:// www.iirrt.ie/wp-content/uploads/2012/02/IV-2014.pdf (accessed 27 September 2017).

10. American College of Radiology. ACR - SPR Practice Parameter for the Use of Intravascular Contrast Media. Reston, VA: ACR, 2014. http://www.acr. org/ /media/536212D711524DA5A4532407082C89BA.pdf (accessed 29 July 2017).

11. University of Malta Department of Radiography. Administration of Prescribed Medicinals by Radiographers. Msida: University of Malta Department of Radiography, 2010. https://www.um.edu.mt/ data/assets/pdf file/0005/85568/IntravenousInjectionsLogbookJuly2010new.pdf (accessed 29 July 2017).
12. Ontario Association of Medical Radiation Sciences. Intravenous Injection of Contrast Media - Competency Profile. Hamilton: OMARS, 2010. https://www. regonline.ca/custlmages/300000/305453/IVInjection_CompetencyProfile.pdf (accessed 29 July 2017).

13. Queensland Department of Health. Medical Imaging Contrast Media (Intravascular). Herston: Queensland Department of Health, 2013. https://www.health qld.gov.au/qhpolicy/docs/gdl/qh-gdl-016.pdf (accessed 29 July 2017).

14. Laerd Statistics. 2013. Descriptive and Inferential Statistics. https://statistics.laerd. com/statistical-guides/descriptive-inferential-statistics.php (accessed 29 July 2017) Derby: Lund Research Ltd., 2013.

15. Tavakol M, Dennick R. Making sense of Cronbach's alpha. Int J Med Educ 2011;2:53 55. https://doi.org/10.5116/ijme.4dfb.8dfd

16. Brink H, van der Walt C, van Rensburg G. Fundamentals of Research Methodology for Healthcare Professionals. 3rd ed. Cape Town: Juta and Company Ltd., 2012.

17. Friedrich-Nel H, Munro L. Radiographers' opinion on patients' rights to informed consent: Results of an online survey. S Afr Radiographer 2015;53(1):27-32.

18. Hall DE, Prochazka AV, Fink AS. Informed consent for clinical treatment. Can Med Ass J 2012;184(5):533-540. https://doi.org/10.1503/cmaj.112120

19. Mathuray M. A critical evaluation of nurses' legal knowledge and its impact in preventing nursing malpractice in South Africa. LLM (Medical Law) thesis. Durban: University of KwaZulu-Natal, 2017.

20. Health Professions Council of South Africa. Guidelines for Good Practice in the Health Care Professions - National Patients' Rights Charter, Booklet 3. Pretoria: HPCSA, 2008. http://www.hpcsa.co.za/downloads/conduct_ethics/rules/generic_ethical_rules/ booklet_3_patients_rights_charter.pdf (accessed 26 September 2017).

21. Royal College of Radiologists. Standards for Intravascular Contrast Agent Administration to Adult Patients. 2nd ed. London: Royal College of Radiologists, 2010. https://www.rcr.ac.uk/sites/default/files/docs/radiology/pdf/BFCR(10)4_Stand_ contrast.pdf (accessed 29 July 2017).

22. Pomara C, Pascale N, Maglietta F, Neri M, Riezzo I, Turillazzi E. Use of contrast media in diagnostic imaging: Medico-legal considerations. La Radiologia Medica 2015;120(9):802-809. https://doi.org/10.1007/s11547-015-0549-6

23. Hesley G, Hartman R. Review of common and uncommon contrast media reactions Applied Radiology. http://appliedradiology.com/articles/review-of-common-anduncommon-contrast-media-reactions (accessed 10 November 2017).

24. Gray A. Prescribing in South Africa: What's Next? Johannesburg: Southern African HIV Clinicians Society, 2014. http://www.sahivsoc.org/Files/Thurs Andy Gray\%20 Prescribing\%20in\%20south\%20africa.pdf (accessed 29 May 2018).

25. Larson SD, Hebra A, Lee S, Raju R. Vascular Access in Children. Medscape, 2014. http://emedicine.medscape.com/article/1018395-overview (accessed 27 September 2017).

26. Peckham C. Medscape Malpractice Report 2015: Why Radiologists Get Sued. Medscape, 2016. http://www.medscape.com/features/slideshow/malpracticereport-2015/radiology\#page $=4$ (accessed 26 September 2017).

27. Lightfoot CB, Abraham RJ, Mammen T, Abdolell M, Kapur S, Abraham RJ. Survey of radiologists' knowledge regarding the management of severe contrast materialinduced allergic reactions. Radiology 2009;251(3):691-696.

28. Rawson JV, Pelletier AL. When to order contrast-enhanced CT. Am Fam Physician 2013;88(5):312-316. http://www.ncbi.nlm.nih.gov/pubmed/24010394 (accessed 28 May 2018).

29. Robbins JB, Pozniak MA. Contrast Media Tutorial. Madison: University of Wisconsin Department of Radiology, 2010. https://www.radiology.wisc.edu/fileShelf/ contrastCorner/ContrastAgentsTutorial.pdf (accessed 27 May 2018).

Accepted 12 June 2018 